\title{
Dynamic Three-Dimensional Analysis of Lachman Test for Anterior Cruciate Ligament Insufficiency: Analysis of Anteroposterior Motion of the Medial and Lateral Femoral Epicondyles
}

Seungbum Koo, $\mathrm{PhD}^{1, \star}$, Bong Soo Kyung, $\mathrm{MD}^{2 \star \star}$, Ju Seon Jeong, $\mathrm{MD}^{3}$, Dong Won Suh, $\mathrm{MD}^{2}$, Jin Hwan Ahn, $\mathrm{MD}^{4}$, and Joon Ho Wang, $\mathrm{MD}^{5}$

${ }^{1}$ School of Mechanical Engineering, Chung-Ang University, Seoul; ${ }^{2}$ Department of Orthopedic Surgery, Barunsesang Hospital, Seongnam, ${ }^{3}$ Department of Orthopedic Surgery, Bumin General Hospital, Busan; ${ }^{4}$ Department of Orthopaedic Surgery, Kangbuk Samsung Medical Center, Seoul; ${ }^{5}$ Department of Orthopaedic Surgery, Samsung Medical Center, Seoul, Korea

\begin{abstract}
Purpose: To evaluate dynamic three-dimensional (3D) kinematic properties of the anterior cruciate ligament (ACL)-insufficient knees and healthy contralateral knees in awake patients during the Lachman test using biplane fluoroscopy.

Materials and Methods: Ten patients with unilateral ACL-insufficient knees and healthy contralateral knees were enrolled in this study. Each patient underwent the Lachman test three times in the awake state. The knee joint motions were captured using biplane fluoroscopy. After manual registration of 3D surface data from 3D-computed tomography to biplane images, dynamic 3D kinematic data were analyzed.

Results: The average anteroposterior (AP) translation of the medial femoral epicondyle of the ACL-insufficient knees (11.5 $\pm 4.2 \mathrm{~mm})$ was significantly greater than that of the contralateral knees $(7.7 \pm 3.5 \mathrm{~mm})(\mathrm{p}<0.05)$. However, there was no statistically significant side-to-side difference in the average AP translation of the lateral femoral epicondyle. During the Lachman test, the distal femur was more externally rotated than the proximal tibia, which showed significant difference between both sides.

Conclusions: During the Lachman test, the medial femoral epicondyle of the ACL-insufficient knee exhibited greater AP motion than that of the contralateral knee, whereas there was no significant side-to-side difference with regard to the AP motion of the lateral femoral epicondyle.
\end{abstract}

Keywords: Anterior cruciate ligament, Anterior cruciate ligament insufficiency, Lachman test, Biplane fluoroscopy, Three-dimensional kinematics

\section{Introduction}

Anterior cruciate ligament (ACL) injury is one of the most

Received December 15, 2014; Revised (1st) March 6, 2015;

(2nd) June 8, 2015; Accepted June 21, 2015

Correspondence to: Joon Ho Wang, MD

Department of Orthopaedic Surgery, Samsung Medical Center, 81

Irwon-ro, Gangnam-gu, Seoul 06351, Korea

Tel: +82-2-3410-3507, Fax: +82-2-3410-0035

E-mail: mdwang88@gmail.com

${ }^{\star}$ The first two authors contributed equally to this study.

This is an Open Access article distributed under the terms of the Creative Commons Attribution Non-Commercial License (http://creativecommons.org/licenses/by-nc/4.0/) which permits unrestricted non-commercial use, distribution, and reproduction in any medium, provided the original work is properly cited. common sports-related injuries of the knee joints ${ }^{1)}$. As ACL injury is closely related to functional disability, knee joint laxity, secondary injuries such as meniscus tear, chondral injury, and post-traumatic osteoarthritis ${ }^{2,3)}$, the importance of diagnosis and treatment of ACL injury cannot be underestimated. For proper screening and diagnosis of ACL insufficiency, physical examination maneuvers such as Lachman test, pivot shift test, and anterior drawer test have been used. Even though some studies have reported a higher sensitivity and specificity of the Lachman test ${ }^{4-6}$, there is still controversy on how to diagnose ACL insufficiency, and some modified methods, such as Lachman test in prone position $^{7)}$, have been introduced and evaluated.

In spite of the long history and presence of many studies on Lachman test, there is a paucity in the literature on the in vivo 
data of three-dimensional (3D) dynamic properties of Lachman test. Some quantitative devices, such as the KT arthrometer or Telos-stress radiography system ${ }^{8}$, have been used to quantify the anterior laxity; however, these devices are designed to assess only two-dimensional anteroposterior (AP) translation. Some studies performed a dynamic 3D analysis of ACL-insufficiency using newly developed tools such as navigation and electromagnetic devices $^{9-13)}$. However, because soft tissue markers have a mismatch between the skin and the bone and bony markers can only be used under anesthesia, these studies only assessed the knee joint under sedation or the cadaveric knees. Two studies performed 3D analysis during Lachman test without anesthesia ${ }^{14,15)}$, but they evaluated only static points: neutral, reverse Lachman and Lachman test using open magnetic resonance imaging $(\mathrm{MRI})^{14)}$; neutral and Lachman test using computed tomography (CT) $\operatorname{scan}^{15)}$.

Biplane fluoroscopy has been used for dynamic 3D analysis of knee joints ${ }^{12,16-19}$. As it can be utilized in in vivo studies without anesthesia and accurately measure the 6 degrees of freedom kinematics, biplane fluoroscopy can be used for dynamic 3D analysis of the joint laxity in the Lachman test. Our literature search revealed that there is no study evaluating the biomechanical properties of the knee joint using biplane fluoroscopy.

The aim of the current study was to evaluate the dynamic 3D kinematic properties of the ACL-insufficient knees and contralateral healthy knees in awake patients during the Lachman test using biplane fluoroscopy. Based on the evaluation, we attempted to determine the efficacy of Lachman test and suggest an appropriate method for improving the reliability of the test.

\section{Materials and Methods}

The study protocol of the current study was approved by the Institutional Review Board of Samsung Medical Center (2011-08075), and all of the participants signed an informed consent form. A total of ten patients with unilateral ACL insufficiency and healthy contralateral knees participated in this prospective study from June 2012 to January 2013. All patients were diagnosed with grade II or III instability in the Lachman test and pivot shift test performed by a single surgeon (Wang), and the ACL insufficiency was confirmed with MRI by the same surgeon and a musculoskeletal radiologist. All the patients were scheduled for ACL reconstruction surgery at our institution. Contralateral knees were also evaluated with MRI and physical examination, and the presence of normal ACL was confirmed. All of the patients were male, their mean age was 34 years, and the mean interval from injury to the test was 2.9 months. Their mean height was $171 \mathrm{~cm}$ and mean weight was $76.7 \mathrm{~kg}$. Eight patients had injuries to the right knee and the other 2 patients had injuries to the left knee. Four patients had meniscus tears in the ACL-insufficient knees: one patient had a peripheral tear of the posterior horn of medial meniscus; two patients had root tears of the posterior horn of lateral meniscus; and one patient had a tear of the posterior horn of medial and lateral menisci. The demographics of participants are shown in Table 1.

\section{Study Protocol and Biplane Fluoroscopic System}

By manual registration of consecutive biplane images during the Lachman test and 3D model obtained from 3D-CT, dynamic 3D data of bilateral knees during the Lachman test were obtained. During the biplane fluoroscopy, each participant underwent the Lachman test three times for bilateral knees in the awake state. For biplane fluoroscopy, the patient lay on the table with a 15$\mathrm{cm}$ knee support placed under the thigh to elicit 30 degree knee flexion. Due to the limited space on the table, the examiner stood beside the lateral aspect of the participant's lower leg and grasped the medial side of proximal tibia of the right knee by the right hand, whereas the examiner's left hand grasped the proximal tibia for the left knee. Another examiner or an assistant grasped the anterior aspect of the thigh by hand to prevent anterior translation of the femur. With the patient placed in this position, the proximal tibia was anteriorly pulled as much as possible. The test was done three times for each leg, and the analysis was done using data obtained from the second test. All the tests were performed by a single orthopedic surgeon, and AP and lateral views were obtained using dynamic biplane fluoroscopy (Artis zee; Siemens, Erlangen, Germany). Before capturing the images, 3D geometric calibration was performed, and then high-resolution serial images were captured in $1440 \times 1440$ pixels at a frame rate of 15/sec. In addition, 3D knee models of all participants were obtained using 3D-CT after ACL reconstruction. Using one CT scanner (Light Speed VCT; GE Healthcare, Little Chalfont, UK),

Table 1. Patient Demographics

\begin{tabular}{lc}
\hline \multicolumn{1}{c}{ Characteristics } & Mean (range) \\
\hline Age $(\mathrm{yr})$ & $34(23-52)$ \\
Sex & Male \\
Height $(\mathrm{cm})$ & $171.0(151.7-179.1)$ \\
Weight $(\mathrm{kg})$ & $76.7(49.7-107)$ \\
Body mass index $\left(\mathrm{kg} / \mathrm{m}^{2}\right)$ & $26.1(21.4-37.1)$ \\
Time from injury to test $(\mathrm{mo})$ & $2.9(1-6)$ \\
\hline
\end{tabular}


axial images of bilateral distal femurs and proximal tibias were obtained with $16 \times 0.625 \mathrm{~mm}$ collimation, $0.625 \mathrm{~mm}$ slice thickness and $512 \times 512$ acquisition matrix. Using Mimics (Materialise, Leuven, Belgium), 100-mm long 3D models of the distal femurs and proximal tibias were created.

The acquired 3D models of the distal femur and proximal tibia were imported into the biplane fluoroscopic system and matched
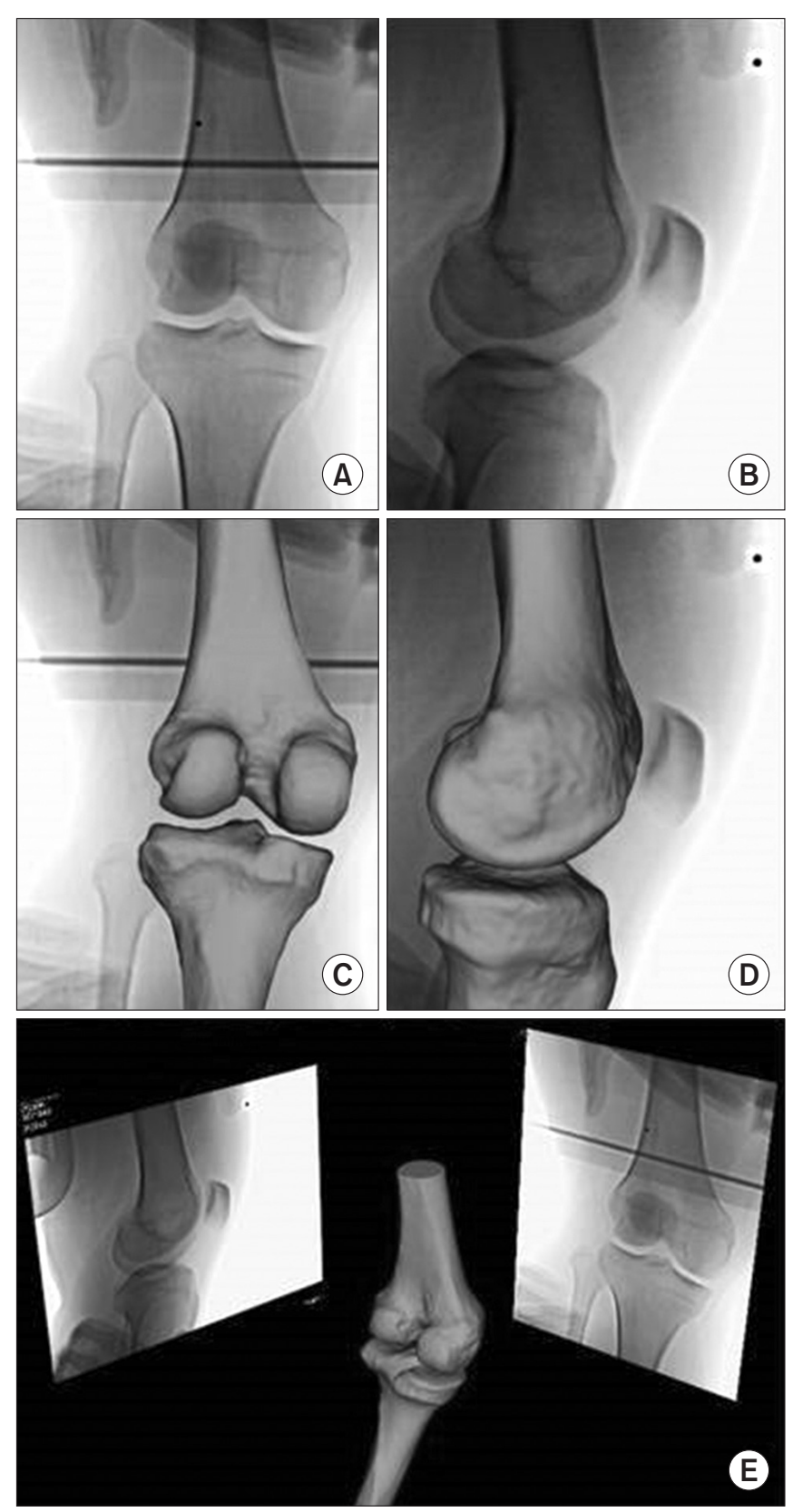

Fig. 1. Manual registration of biplane fluoroscopic images and threedimensional (3D) model. (A, B) Two-dimensional dynamic anteroposterior and lateral images were obtained by biplane fluoroscopy. (C, D) All of the images were matched by manual segmentation with patient's $3 \mathrm{D}$ knee joint model. (E) Acquired 3D dynamic model and data. by manual registration. One of the graduate students in the biomechanical engineering laboratory manually matched the $3 \mathrm{D}$ model to the fluoroscopic image (Fig. 1).

\section{Analysis Method Using a New Coordinate System}

For the quantitative analysis and comparison of 3D motions between the distal femur and proximal tibia, the motion of the distal femur relative to the fixed proximal tibia was assessed using a new coordinate system that was created using anatomical references of the proximal tibia. The 3D model of the proximal tibia was imported into Geomagic 2012 (Research Triangle Park, North Carolina, USA), and the surface of the medial tibial plateau was selected. Using the 'adjustment tool' in Geomagic 2012 for the selected surface, the plane of medial tibial plateau was established and defined as the $x y$ plane ( $x$ axis represents anteriorposterior, $y$ axis represents medial-lateral, $z$ axis represents proximal-distal). In addition, we defined the AP direction by using two anatomical landmarks of the proximal tibia, medial 1/3rd of the tibial tuberosity and the center of posterior cruciate ligament insertion $^{20,21)}$ (Fig. 2). Using these definitions of the axes, a new coordinate system was created and this coordinate system was applied to the obtained dynamic 3D data during the tests. Using a mathematics software package (Matlab; MathWorks Inc., Natick, MA, USA), we checked AP translation of the two femoral landmarks, medial femoral epicondyle and lateral femoral epicondyle, and axial rotation of the distal femur (Fig. 3).

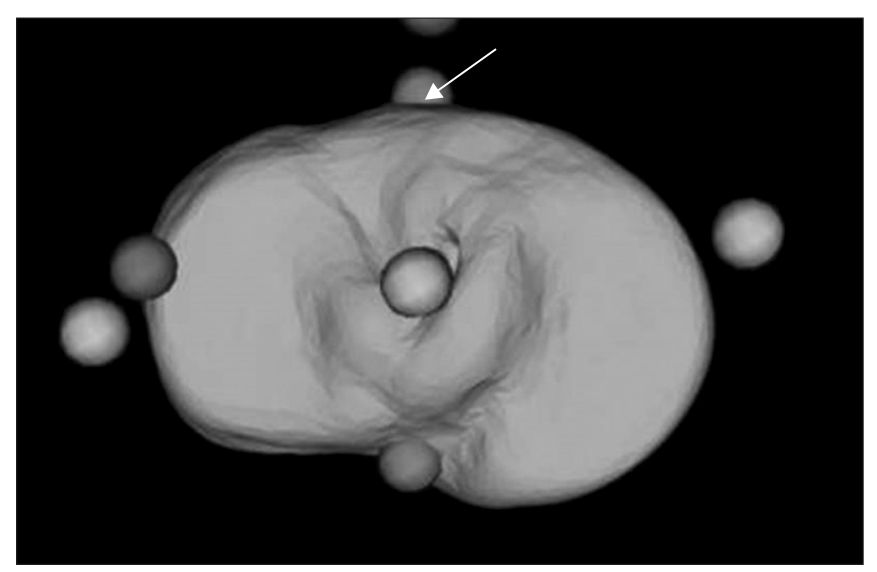

Fig. 2. New coordinate system for the proximal tibia. Using anatomical landmarks of the proximal tibia, new $x, y, z$ axes were established. The $x y$ plane was defined as the surface of medial tibial plateau, and the anteroposterior axis was defined as the line between the medial $1 / 3$ of the tibial tuberosity and center of posterior cruicate ligament (PCL) insertion. The arrow indicates the medial $1 / 3$ of the tibial tuberosity and the center of PCL insertion. 

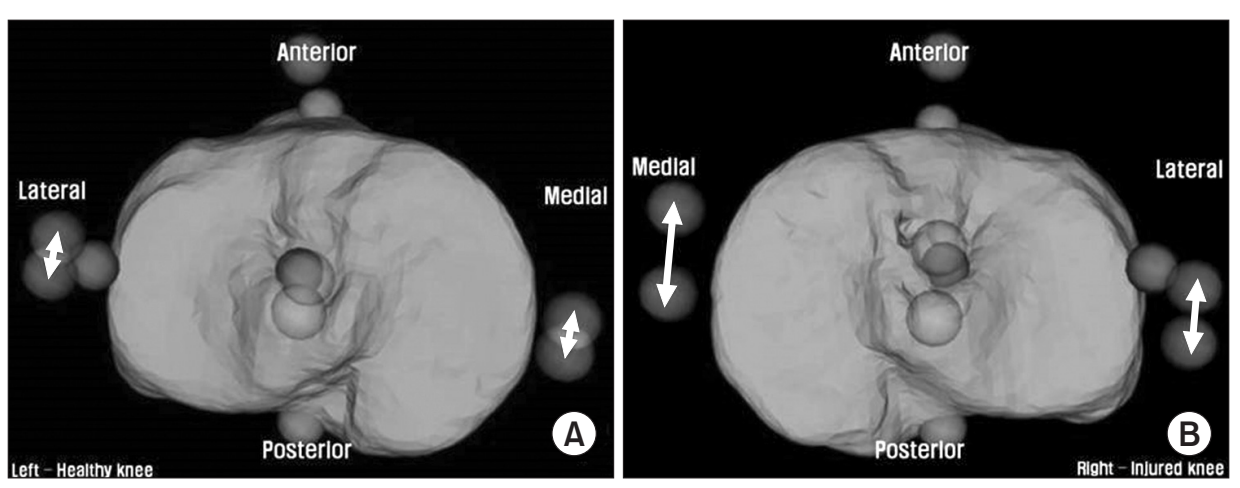

Fig. 3. A 23-year-old male patient with anterior cruciate ligament (ACL) insufficiency in the right knee showed increased anteroposterior (AP) translation of the medial femoral epicondyle in the ACL insufficient knee (B) than that in the healthy control knee (A). However, there was no statistically significant difference in the average AP translation of the lateral femoral epicondyle between the ACL-insufficient knee and contralateral knee.

\section{Statistical Analysis}

We compared AP translation of the medial and lateral femoral epicondyles during the Lachman test between ACL-insufficient knees and the contralateral healthy knees. For statistical analysis, Mann-Whitney test was used to compare the AP translation between ACL-insufficient knees and healthy knees. A p-value of less than 0.05 was considered statistically significant, and statistical analysis was performed using SPSS ver. 12.0 (SPSS Inc., Chicago, IL, USA).

\section{Results}

The mean AP translation of the medial femoral epicondyle of the ACL-insufficient knees was $11.5 \pm 4.2 \mathrm{~mm}$ (range, 7.1 to 19.6 $\mathrm{mm}$ ) and that of the lateral femoral epicondyle of the ACL-insufficient knees was $11.8 \pm 3.5 \mathrm{~mm}$ (range, 7.1 to $17.4 \mathrm{~mm}$ ). In the contralateral healthy knees, the mean AP translation of the medial femoral epicondyle was $7.7 \pm 3.5 \mathrm{~mm}$ (range, 3.7 to $14.5 \mathrm{~mm}$ ) and that of the lateral femoral epicondyle was $8.8 \pm 3.3 \mathrm{~mm}$ (range, 3.7 to $13.5 \mathrm{~mm}$ ). On statistical analysis, the mean AP translation of the medial femoral epicondyle was significantly different between the ACL-insufficient knees and contralateral knees $(\mathrm{p}<0.05$, Table 2); however, the mean AP translation of the lateral femoral epicondyle was not significantly different between the ACLinsufficient knees and contralateral knees ( $p=0.089$, Table 2).

On axial rotation during the Lachman test, the mean rotation of the ACL-insufficient knees was 3.0 \pm 2.4 degrees (range, 0 to 6.5 degree) and that of the contralateral healthy knees was $2.4 \pm 2.3$ degrees (range, 0 to 6.5 degree). All of the knees showed tibial internal rotation during the test, and there was no statistically significant difference between ACL-insufficient knees and contralateral healthy knees ( $\mathrm{p}=0.835$, Table 2 ).

Translations at both epicondyles and axial ration in all testes knees are shown in Table 3.
Table 2. Mean Anteroposterior (AP) Translation and Axial Rotation

\begin{tabular}{cccc}
\hline Variable & $\begin{array}{c}\text { ACL-insufficient } \\
\text { knee }\end{array}$ & Control & p-value \\
\hline AP translation $(\mathrm{mm})$ & & & \\
Medial femoral epicondyle & $11.5 \pm 4.2$ & $7.7 \pm 3.5$ & 0.035 \\
Lateral femoral epicondyle & $11.8 \pm 3.5$ & $8.8 \pm 3.3$ & 0.089 \\
Axial rotation $\left({ }^{\circ}\right)$ & $3.0 \pm 2.4$ & $2.4 \pm 2.3$ & 0.853 \\
\hline
\end{tabular}

Values are presented as mean \pm standard deviation.

ACL: anterior cruciate ligament.

${ }^{a)} \mathrm{p}<0.05$ is considered statistically significant.

\section{Discussion}

The purpose of the current study was to evaluate $3 \mathrm{D}$ dynamic data obtained during the Lachman test in awake patients and to identify the most important factor in the diagnosis of ACL insufficiency. According to the current study, the average AP translation of the medial femoral epicondyle showed a more significant difference than the average AP translation of the lateral femoral epicondyle between ACL-insufficient knees and healthy contralateral knees. This indicates that the medial side has more AP motion in ACL-insufficient knees and the physicians should focus on the motion of the medial femoral condyle for making a diagnosis of ACL insufficiency using the Lachman test.

The current study focused on realizing the in vivo situation during Lachman test in clinics. Various studies have assessed kinematics of ACL-insufficient knees during the Lachman test ${ }^{9-11,16-18,22-25)}$. However, considering that our study evaluated data obtained from the awake patients during the test, we believe our results would be the more similar to those in a real clinical setting where the test results are influenced by the muscles and tendons around the knee joint. In order to evaluate the motion during Lachman test in a real clinical setting, in vivo studies without anesthesia are necessary. Logan et al. ${ }^{14)}$ evaluated the Lachman test results of awake participants. The study evaluated the three static points; 
Table 3. Results

\begin{tabular}{|c|c|c|c|c|c|c|c|c|c|c|}
\hline \multirow[b]{3}{*}{ No. } & \multirow{3}{*}{$\begin{array}{l}\text { Age } \\
(\mathrm{yr})\end{array}$} & \multirow[b]{3}{*}{ Sex } & \multirow{3}{*}{$\begin{array}{l}\text { Injured } \\
\text { side }\end{array}$} & \multirow{3}{*}{$\begin{array}{l}\text { Interval from } \\
\text { injury to test } \\
\quad(\mathrm{mo})\end{array}$} & \multicolumn{6}{|c|}{ AP translation during Lachman test } \\
\hline & & & & & \multicolumn{3}{|c|}{ ACL-insufficient knee } & \multicolumn{3}{|c|}{ Healthy contralateral knee } \\
\hline & & & & & $\begin{array}{c}\text { Medial } \\
\text { epicondyle } \\
(\mathrm{mm})\end{array}$ & $\begin{array}{c}\text { Lateral } \\
\text { epicondyle } \\
(\mathrm{mm})\end{array}$ & $\begin{array}{c}\text { Rotation } \\
\left({ }^{\circ}\right)\end{array}$ & $\begin{array}{l}\text { Medial } \\
\text { epicondyle } \\
(\mathrm{mm})\end{array}$ & $\begin{array}{c}\text { Lateral } \\
\text { epicondyle } \\
(\mathrm{mm})\end{array}$ & $\begin{array}{c}\text { Rotation } \\
\quad\left({ }^{\circ}\right)\end{array}$ \\
\hline 1 & 52 & M & $\mathrm{L}$ & 3.5 & 9.0 & 17.4 & 4.9 & 14.5 & 13.5 & 6.5 \\
\hline 2 & 38 & M & $\mathrm{R}$ & 2.5 & 14.5 & 13.5 & 6.5 & 9.5 & 8.5 & 4.1 \\
\hline 3 & 28 & M & $\mathrm{L}$ & 1 & 7.1 & 7.1 & 0 & 4.3 & 4.3 & 0 \\
\hline 4 & 23 & M & $\mathrm{R}$ & 3 & 19.6 & 10.8 & 4.1 & 9.4 & 8.7 & 5.0 \\
\hline 5 & 36 & M & $\mathrm{R}$ & 5 & 13.3 & 14.6 & 5.4 & 6.2 & 8.2 & 1.4 \\
\hline 6 & 23 & M & $\mathrm{R}$ & 2 & 15.5 & 11.6 & 2.5 & 6.8 & 6.3 & 1.3 \\
\hline 7 & 33 & M & $\mathrm{R}$ & 3 & 7.2 & 7.2 & 0 & 12.0 & 12.0 & 0 \\
\hline 8 & 23 & M & $\mathrm{R}$ & 1 & 9.1 & 9.1 & 0 & 3.7 & 3.7 & 0 \\
\hline 9 & 43 & M & $\mathrm{R}$ & 2 & 7.9 & 15.3 & 4.6 & 4.7 & 10.5 & 2.9 \\
\hline 10 & 43 & $\mathrm{M}$ & $\mathrm{R}$ & 6 & 12.3 & 11.3 & 2.4 & 6.2 & 11.8 & 2.6 \\
\hline
\end{tabular}

AP: anteroposterior, ACL: anterior cruciate ligament, L: left, R: right.

reverse Lachman, neutral and Lachman, using open MRI. They showed medial and lateral sides of ACL-insufficient knees had more significant increase during Lachman test than normal knees. The results were different from ours. This may be attributable to longer time for MRI scanning in the study: ten to twenty minutes were taken for MRI and anterior force during Lachman could have accumulated in the meantime, resulting in greater AP motion of the medial and lateral sides. In contrast, the data in the current study was obtained during the real Lachman test (maybe one to two seconds for anterior drawing), thus there was no accumulation of force.

In previous studies on ACL insufficiency ${ }^{26,277}$, the medial condyle exhibited greater AP motion than the lateral condyle in various situations. It has been known that the lateral femoral condyle undergoes greater AP translation than the medial femoral condyle in normal knees. Komistek et al ${ }^{26)}$ reported that the lateral femoral condyle undergoes more motion than the medial femoral condyle during daily weight-bearing activities ${ }^{26)}$. Dennis et al. ${ }^{27)}$ also reported that the average posterior translation of the lateral femoral condyle was $21 \mathrm{~mm}$ and that of the medial femoral condyle was $2 \mathrm{~mm}$ during knee flexion. The results of the current study were similar to those of the abovementioned studies. In healthy knees, the lateral femoral epicondyle exhibited greater AP motion than the medial femoral epicondyle during the Lachman test. The AP motion of the medial femoral epicondyle of the ACL-insufficient knee joints increased significantly. The AP motion of the lateral femoral epicondyle of ACL-insufficient knee joints also increased, but it was not statistically significant. Den- nis et al. ${ }^{27)}$ also showed similar results in a previous study, which demonstrated that ACL-deficient knees experienced considerable changes in the position of the medial condyle during deep flexion. As the Lachman test, used to evaluate anterior instability in ACL-insufficient knees, was performed in awake patients in our study, it is reasonable to assume that the results of the current study more reliably demonstrate the AP instability of both femoral condyles. The results also show that the ACL may be closely related to the AP motion of the medial femoral condyle than the lateral femoral condyle even though the femoral attachment site of the ACL is on the lateral femoral condyle.

Many meta-analysis studies have reported on the accuracy and reliability of the Lachman test ${ }^{4-6,28}$. The accuracy of the Lachman test varies in different studies, ranging from $63 \%$ to $93 \%$. These studies attempted to demonstrate the statistical significance of the common protocol of the Lachman test, but they did not determine the method to improve the accuracy of the Lachman test. According to the current study, the medial compartment undergoes a significant AP motion during the Lachman test than does the lateral compartment. Thus, we suggest, by focusing on the medial compartment during the Lachman test, the examiner can make an accurate diagnosis of anterior laxity in ACL-insufficient knees.

There are some limitations to the current study. First, this study is limited by the small sample size. Although only ten participants were enrolled in the current study, the statistical analysis showed a significant difference in AP translation of the medial femoral epicondyle and it did not show a significant difference 
in AP translation of the lateral femoral epicondyle. This provides a sufficient basis to conclude that the medial femoral epicondyle motion is more reliable than the lateral femoral epicondyle motion in the diagnosis of ACL-insufficiency. Second, some of the patients had accompanying tears of the meniscus, which is known as the secondary stabilizer of knee joint ${ }^{29,30)}$. Two patients had accompanying peripheral tears of the posterior horn of medial meniscus, and these participants showed greater AP motion of the medial femoral epicondyle, i.e., 8 to $10 \mathrm{~mm}$. Third, the AP translation increased only 2 to $3 \mathrm{~mm}$ in the ACL insufficient knee, indicating less than grade II laxity. Because current study was performed in a limited space of biplane fluoroscopy, participants could feel more discomfort and that could have resulted in limited AP translation. Fourth, evaluation of muscle contracture was not performed in the patients. The strong point of this study is that the study design simulated the real clinical situation during Lachman test; however, muscle contracture around the knee joint, which may affect the AP motion and rotation during the Lachman test, was not assessed.

\section{Conclusions}

The medial femoral epicondyle of the ACL-insufficient knee exhibited greater AP motion than that of the contralateral knee during the Lachman test. In contrast, the lateral femoral epicondyle of the ACL-insufficient knee did not exhibit greater AP motion than that of the contralateral knee.

\section{Conflict of Interest}

No potential conflict of interest relevant to this article was reported.

\section{Acknowledgements}

This research was supported by Basic Science Research Program through the National Research Foundation of Korea (NRF) funded by the Ministry of Education, Science and Technology (No. NRF-2012R1A1A2043793). The study protocol of the current study was approved by the Institutional Review Board at Samsung Medical Center (2011-08-075).

\section{References}

1. Gianotti SM, Marshall SW, Hume PA, Bunt L. Incidence of anterior cruciate ligament injury and other knee ligament injuries: a national population-based study. J Sci Med Sport. 2009;12:622-7.

2. Allen CR, Livesay GA, Wong EK, Woo SL. Injury and reconstruction of the anterior cruciate ligament and knee osteoarthritis. Osteoarthritis Cartilage. 1999;7:110-21.

3. Oiestad BE, Engebretsen L, Storheim K, Risberg MA. Knee osteoarthritis after anterior cruciate ligament injury: a systematic review. Am J Sports Med. 2009;37:1434-43.

4. Benjaminse A, Gokeler A, van der Schans CP. Clinical diagnosis of an anterior cruciate ligament rupture: a metaanalysis. J Orthop Sports Phys Ther. 2006;36:267-88.

5. Scholten RJ, Opstelten W, van der Plas CG, Bijl D, Deville WL, Bouter LM. Accuracy of physical diagnostic tests for assessing ruptures of the anterior cruciate ligament: a metaanalysis. J Fam Pract. 2003;52:689-94.

6. Ostrowski JA. Accuracy of 3 diagnostic tests for anterior cruciate ligament tears. J Athl Train. 2006;41:120-1.

7. Mulligan EP, Harwell JL, Robertson WJ. Reliability and diagnostic accuracy of the Lachman test performed in a prone position. J Orthop Sports Phys Ther. 2011;41:749-57.

8. Tyler TF, McHugh MP, Gleim GW, Nicholas SJ. Association of KT-1000 measurements with clinical tests of knee stability 1 year following anterior cruciate ligament reconstruction. J Orthop Sports Phys Ther. 1999;29:540-5.

9. Robinson J, Carrat L, Granchi C, Colombet P. Influence of anterior cruciate ligament bundles on knee kinematics: clinical assessment using computer-assisted navigation. Am J Sports Med. 2007;35:2006-13.

10. Colombet P. Knee laxity control in revision anterior cruciate ligament reconstruction versus anterior cruciate ligament reconstruction and lateral tenodesis: clinical assessment using computer-assisted navigation. Am J Sports Med. 2011; 39:1248-54.

11. Araki D, Kuroda R, Matsushita T, Matsumoto T, Kubo S, Nagamune K, Kurosaka M. Biomechanical analysis of the knee with partial anterior cruciate ligament disruption: quantitative evaluation using an electromagnetic measurement system. Arthroscopy. 2013;29:1053-62.

12. Ahlden M, Hoshino Y, Samuelsson K, Araujo P, Musahl V, Karlsson J. Dynamic knee laxity measurement devices. Knee Surg Sports Traumatol Arthrosc. 2012;20:621-32.

13. Kuroda R, Hoshino Y, Kubo S, Araki D, Oka S, Nagamune K, Kurosaka M. Similarities and differences of diagnostic manual tests for anterior cruciate ligament insufficiency: a global survey and kinematics assessment. Am J Sports Med. 2012;40:91-9. 
14. Logan MC, Williams A, Lavelle J, Gedroyc W, Freeman M. What really happens during the Lachman test? A dynamic MRI analysis of tibiofemoral motion. Am J Sports Med. 2004;32:369-75.

15. Nordt WE 3rd, Lotfi P, Plotkin E, Williamson B. The in vivo assessment of tibial motion in the transverse plane in anterior cruciate ligament-reconstructed knees. Am J Sports Med. 1999;27:611-6.

16. Brandsson S, Karlsson J, Eriksson BI, Karrholm J. Kinematics after tear in the anterior cruciate ligament: dynamic bilateral radiostereometric studies in 11 patients. Acta Orthop Scand. 2001;72:372-8.

17. Brandsson S, Karlsson J, Sward L, Kartus J, Eriksson BI, Karrholm J. Kinematics and laxity of the knee joint after anterior cruciate ligament reconstruction: pre- and postoperative radiostereometric studies. Am J Sports Med. 2002;30:361-7.

18. Defrate LE, Papannagari R, Gill TJ, Moses JM, Pathare NP, Li G. The 6 degrees of freedom kinematics of the knee after anterior cruciate ligament deficiency: an in vivo imaging analysis. Am J Sports Med. 2006;34:1240-6.

19. Tashman S, Collon D, Anderson K, Kolowich P, Anderst W. Abnormal rotational knee motion during running after anterior cruciate ligament reconstruction. Am J Sports Med. 2004;32:975-83.

20. Lutzner J, Krummenauer F, Gunther KP, Kirschner S. Rotational alignment of the tibial component in total knee arthroplasty is better at the medial third of tibial tuberosity than at the medial border. BMC Musculoskelet Disord. 2010; 11:57.

21. Kawahara S, Matsuda S, Okazaki K, Tashiro Y, Mitsuyasu H, Nakahara H, Iwamoto Y. Relationship between the tibial anteroposterior axis and the surgical epicondylar axis in varus and valgus knees. Knee Surg Sports Traumatol Arthrosc. 2012;20:2077-81.

22. Lerat JL, Moyen BL, Cladiere F, Besse JL, Abidi H. Knee in- stability after injury to the anterior cruciate ligament. Quantification of the Lachman test. J Bone Joint Surg Br. 2000; 82:42-7.

23. Hurley WL, Boros RL, Challis JH. Influences of variation in force application on tibial displacement and strain in the anterior cruciate ligament during the Lachman test. Clin Biomech (Bristol, Avon). 2004;19:95-8.

24. Maeda S, Ishibashi Y, Tsuda E, Yamamoto Y, Toh S. Intraoperative navigation evaluation of tibial translation after resection of anterior cruciate ligament remnants. Arthroscopy. 2011;27:1203-10.

25. Christel PS, Akgun U, Yasar T, Karahan M, Demirel B. The contribution of each anterior cruciate ligament bundle to the Lachman test: a cadaver investigation. J Bone Joint Surg Br. 2012;94:68-74.

26. Komistek RD, Dennis DA, Mahfouz M. In vivo fluoroscopic analysis of the normal human knee. Clin Orthop Relat Res. 2003;(410):69-81.

27. Dennis DA, Mahfouz MR, Komistek RD, Hoff W. In vivo determination of normal and anterior cruciate ligamentdeficient knee kinematics. J Biomech. 2005;38:241-53.

28. van Eck CF, van den Bekerom MP, Fu FH, Poolman RW, Kerkhoffs GM. Methods to diagnose acute anterior cruciate ligament rupture: a meta-analysis of physical examinations with and without anaesthesia. Knee Surg Sports Traumatol Arthrosc. 2013;21:1895-903.

29. Ahn JH, Bae TS, Kang KS, Kang SY, Lee SH. Longitudinal tear of the medial meniscus posterior horn in the anterior cruciate ligament-deficient knee significantly influences anterior stability. Am J Sports Med. 2011;39:2187-93.

30. Seon JK, Gadikota HR, Kozanek M, Oh LS, Gill TJ, Li G. The effect of anterior cruciate ligament reconstruction on kinematics of the knee with combined anterior cruciate ligament injury and subtotal medial meniscectomy: an in vitro robotic investigation. Arthroscopy. 2009;25:123-30. 